\title{
Temporal Trends of Sex Disparity in Incidence and Survival of Colorectal Cancer: Variations by Anatomical Site and Age at Diagnosis
}

This article was published in the following Dove Press journal: Clinical Epidemiology

\author{
Ming Sun ${ }^{1,2}$ \\ Youxin Wang $\mathbb{D}^{2}$ \\ Jan Sundquist $\mathbb{D}^{1,3,4}$ \\ Kristina Sundquist ${ }^{1,3,4}$ \\ Jianguang Ji $\mathbb{D}^{\prime}$ \\ 'Center for Primary Health Care \\ Research, Lund University/Region Skåne, \\ Malmö, Sweden; ${ }^{2}$ Beijing Key Laboratory \\ of Clinical Epidemiology, School of Public \\ Health, Capital Medical University, \\ Beijing, People's Republic of China; \\ ${ }^{3}$ Department of Family Medicine and \\ Community Health, Department of \\ Population Health Science and Policy, \\ Icahn School of Medicine at Mount Sinai, \\ New York, NY, USA; ${ }^{4}$ Center for \\ Community-Based Healthcare Research \\ and Education (CoHRE), Department of \\ Functional Pathology, School of Medicine, \\ Shimane University, Shimane, Japan
}

Correspondence: Jianguang Ji Center for Primary Health Care Research, Jan Waldenströms Gata 35, Skåne University Hospital, Malmö 205 02, Sweden

Tel +4640391382

Fax +46 40391370

Email jianguang.ji@med.lu.se

Youxin Wang

Beijing Key Laboratory of Clinical

Epidemiology, School of Public Health,

Capital Medical University, 10 Youanmen

Xitoutiao, Beijing 100069, People's

Republic of China

Tel +86 I0 839I 1779

Fax +86 I0 839। I50।

Email wangy@ccmu.edu.cn
Purpose: The incidence of colorectal cancer (CRC) varies by age, sex, and anatomical subsite. Few studies have examined the temporal trends of age-specific sex disparity in incidence and survival by age at diagnosis and anatomical site.

Patients and Methods: The study was performed on all incident cases of CRC, using data derived from the nationwide Swedish Cancer Register between 1960 and 2014, including right-sided colon cancer (RCC), left-sided colon cancer (LCC), and rectal cancer. Male-tofemale age-standardized incidence rate ratio (IRR) and male-to-female five-year survival rate ratio (SRR) were calculated as the main indicators. Furthermore, we performed joinpoint regression analyses to estimate average annual percentage change.

Results: The overall male-to-female IRR was 1.05 for RCC, 1.31 for LCC, and 1.66 for rectal cancer. Male-to-female IRR increased steadily for RCC by an average of $0.4 \%$ per year until the mid-1990s and then decreased gradually by an average of $1.0 \%$ per year. LCC patients showed an increase of $0.6 \%$ per year since the mid-1970s. For rectal cancer, a non-significant random fluctuation was noted during the study period. The temporal trends of male-to-female IRR varied by age at diagnosis. The male-to-female SRR was 0.87 for RCC, 0.88 for LCC, and 0.86 for rectal cancer, which remained relatively stable during the study period.

Conclusion: Sex disparity of CRC is age-, period-, and anatomical subsite-dependent. Further studies are needed to investigate the underlying contributing factors.

Keywords: colorectal cancer, sex disparity, incidence, survival, temporal trend

\section{Introduction}

Colorectal cancer (CRC) is the third most commonly diagnosed malignancy with more than 1.8 million new cases and the second leading cause of cancer death with more than 800,000 deaths worldwide in 2018. ${ }^{1}$ CRC shows sex differences in incidence and survival. Women are less likely to develop CRC than men, ${ }^{2}$ and women with $\mathrm{CRC}$ have a longer survival time than men. ${ }^{3-5} \mathrm{CRC}$ can be divided into three subsites according to the anatomical areas, including proximal or rightsided colon cancer (RCC), distal or left-sided colon cancer (LCC), and rectal cancer. RCC consists of cancers of the caecum, ascending and transverse colon, and splenic flexure, whereas LCC consists of cancers of the descending and sigmoid colon. A growing amount of data suggest that carcinomas of the right and left colon should be considered as different tumor entities. Meanwhile, sex disparity of CRC varied by anatomical subsite with females being more prone to develop RCC and males being more likely to develop LCC and rectal cancer. ${ }^{2,6}$ 
Although the underlying mechanisms for sex disparity of CRC are still unclear, some previous studies have suggested that the differences between males and females in environmental exposures, molecular pathways, and genetic and epigenetic alterations might contribute to the observed difference. ${ }^{7-10}$ As molecular pathways and genetic and epigenetic alterations are not associated with temporal changes, studies on the temporal trends of sex disparity in CRC and its subsites might provide additional evidence about environmental factors on the development of CRC, which is still largely unknown.

To better understand the sex-specific incidence and survival of CRC during recent decades, our study utilized data derived from the nationwide Swedish Cancer Register to describe the overall and age-specific temporal trends of maleto-female age-standardized incidence rate ratios (IRR) and male-to-female age-standardized five-year survival rate ratios (SRR) of RCC, LCC, and rectal cancer between 1960 and 2014. Furthermore, we performed joinpoint regression analyses to identify potential change points over the study period and to estimate the magnitude of the trends at each time segment.

\section{Materials and Methods Study Population}

The cohort study used data from the Swedish Cancer Register, which was founded in 1958, and has almost complete nationwide coverage. ${ }^{11}$ We identified all diagnoses of CRC according to the $7^{\text {th }}$ Revision of International Classification of Diseases (ICD-7 code) as used in the Swedish Cancer Register. All subsite-, age-, and sex-specific data were extracted from January 1, 1960 to December 31, 2014. Only the first diagnosis of CRC was counted as an incident case, and patients with multiple sites of CRC were not included. For RCC, the codes were 1530 (caecum and ascending colon) and 1531 (transverse colon and the splenic flexure). For LCC the codes were 1532 (descending colon) and 1533 (sigmoid colon). The codes were 1540 and 1548 for rectal cancer.

This study was approved on February 6, 2013 by the Ethics Committee at Lund University, Sweden (ref 2012/ 795). The project database is located at Center for Primary Health Care in Malmö, Sweden.

\section{Statistical Analysis}

Age-standardized sex-specific incidence rate (AIR) and male-to-female IRR were used as the main incidence indicators. The AIR was calculated using the direct method and based on the standard age distribution of the Swedish population in the year 2000. Temporal trends of male-tofemale IRR were plotted according to the year at diagnosis and calculated separately using five-year time intervals: 1960-64, 1965-69, 1970-74, 1975-79, 1980-84, 1985-89, 1990-94, 1995-99, 2000-2004, 2005-2009, and 2010-2014.

Age-standardized sex-specific five-year survival rate (ASR) and male-to-female SRR were used as the main survival indicators. The overall survival analyses were restricted to the period 1960-2009 to ensure all patients with CRC could be followed for more than five years. Survival was counted from the date of diagnosis until the date of death (from any cause) or end of follow-up (December 31, 2014), whichever occurred first.

The trends of male-to-female IRR and male-to-female SRR were evaluated using the joinpoint regression analyses. Analyses were performed using the log-linear model and allowed for a maximum of four joinpoints. Permutation Test was used to select the final model. The annual percent change (APC) was calculated from the slope of the loglinear model. Empirical Quantile method was used to calculate the $95 \%$ confidence interval. We further stratified the analysis by age at onset of CRC: $0-49$ years, and 50 years or older. Data analyses were performed using Joinpoint Regression Program version 4.7.0.0 and SAS version 9.4 (SAS Institute, Cary, NC, USA).

The data that support the findings of this study are available upon request from the corresponding author. The data are not publicly available due to privacy or ethical restrictions.

\section{Results}

During the study period, a total of 66,229 patients were diagnosed with RCC (30,036 [45.4\%] men and 36,193 [54.6\%] women), 52,153 patients with LCC $(27,026$ [51.8\%] men and 25,127 [48.2\%] women), and 76,628 patients with rectal cancer $(44,201$ [57.7\%] men and 32,427 [42.3\%] women).

Table 1 presents the overall AIR and the temporal trends of male-to-female IRR of CRC by anatomical subsites. The AIR of RCC in males was consistently higher than in females until 2004 and then reversed. The lowest male-tofemale IRR of RCC was 0.96 between 2010 and 2014 and the highest male-to-female IRR was 1.11 between 1990 and 1994. The AIR of LCC and rectal cancer in males was consistently higher than females throughout the study period. For LCC, the lowest male-to-female IRR was 1.19 


\begin{tabular}{|c|c|c|c|c|c|}
\hline & \multicolumn{2}{|c|}{ 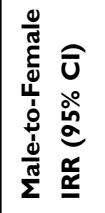 } & 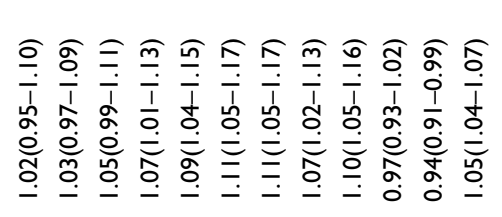 & 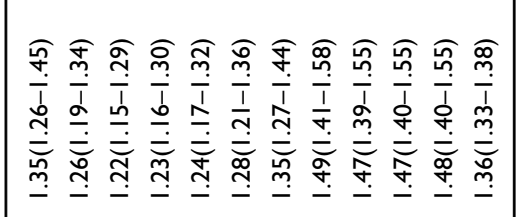 & 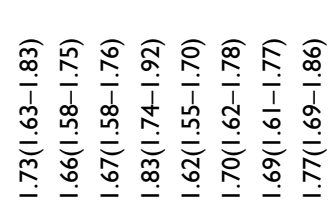 \\
\hline & \multirow[b]{4}{*}{ 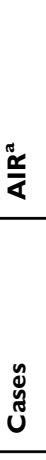 } & 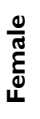 & 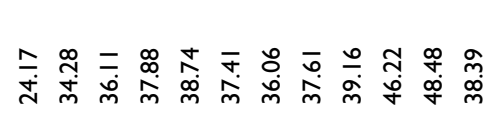 & 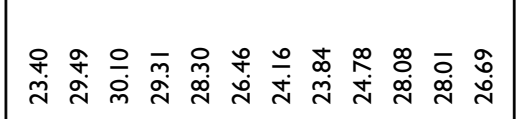 & 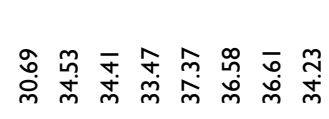 \\
\hline & & $\frac{\frac{0}{\pi}}{\Sigma}$ & 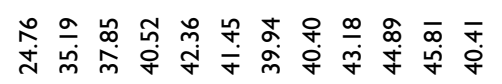 & 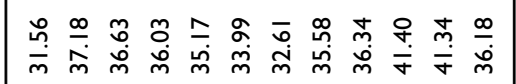 & 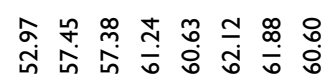 \\
\hline & & 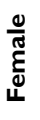 & o్ & 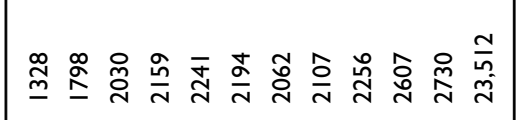 & 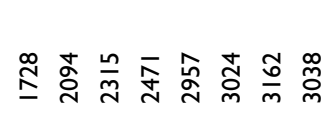 \\
\hline & & $\frac{0}{\frac{\pi}{\Sigma}}$ & 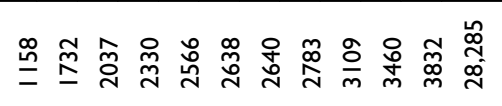 & 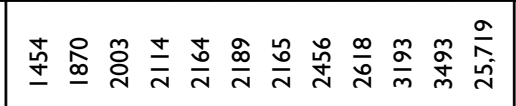 & 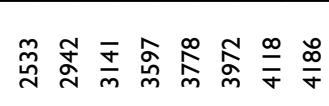 \\
\hline \multicolumn{3}{|c|}{ 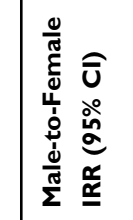 } & 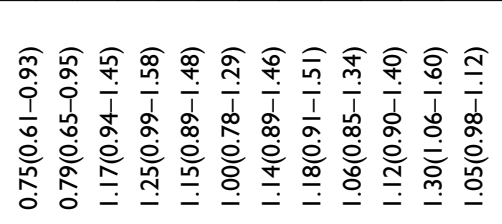 & 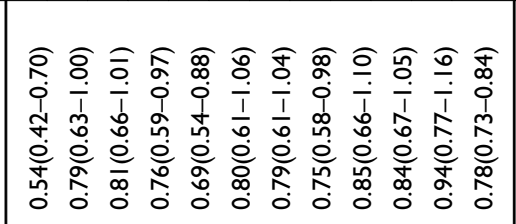 & 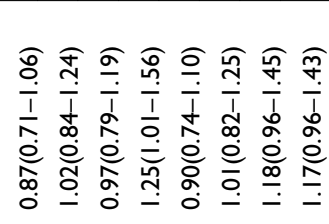 \\
\hline \multirow{4}{*}{ 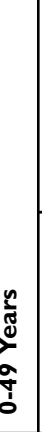 } & \multirow[b]{4}{*}{ ֻू } & $\begin{array}{l}\frac{0}{\pi} \\
\text { है } \\
\text { है }\end{array}$ & 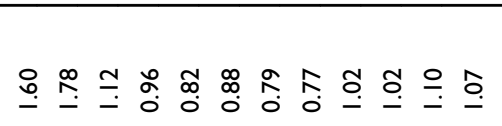 & 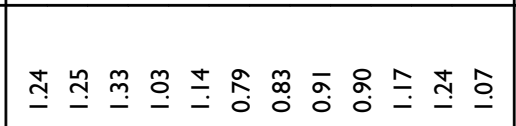 & 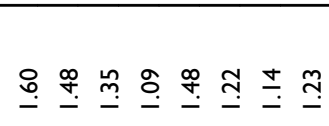 \\
\hline & & $\frac{\frac{0}{\pi}}{\Sigma}$ & 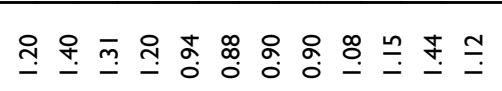 & 必 & 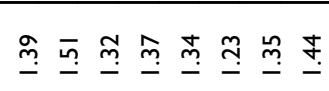 \\
\hline & & 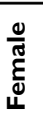 & 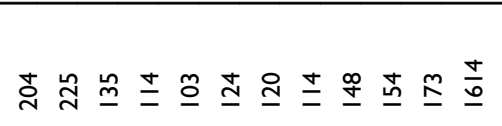 & 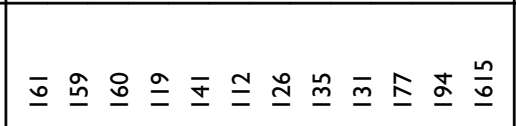 & 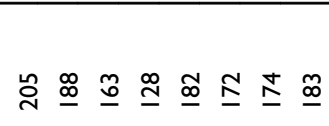 \\
\hline & & $\frac{0}{\frac{0}{\pi}}$ & 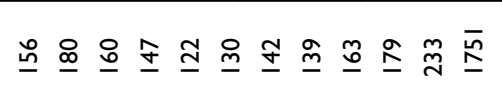 & 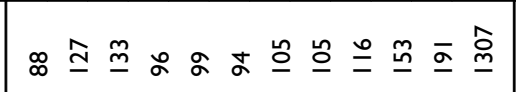 & 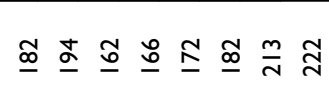 \\
\hline \multicolumn{3}{|c|}{ 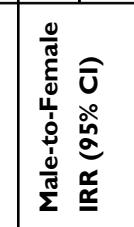 } & 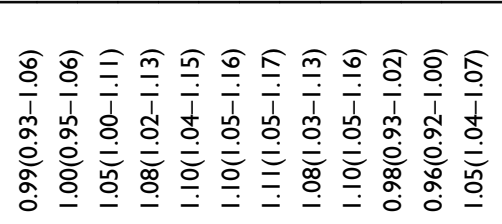 & 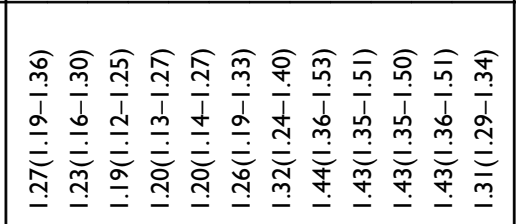 & 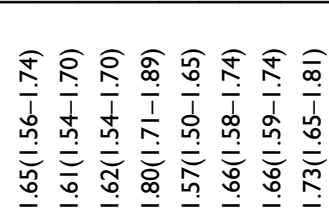 \\
\hline \multirow[b]{4}{*}{ 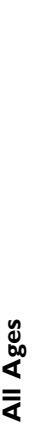 } & \multirow[b]{4}{*}{ 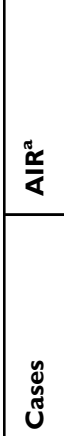 } & 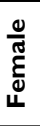 & 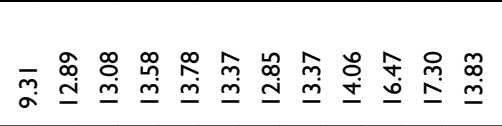 & 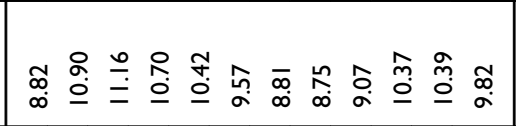 & 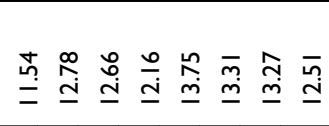 \\
\hline & & $\frac{\frac{0}{\sigma}}{\Sigma}$ & 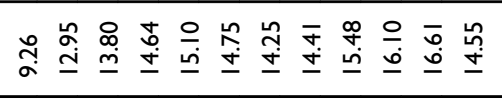 & 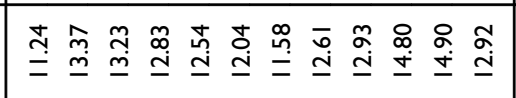 & 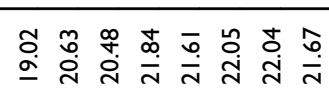 \\
\hline & & $\begin{array}{l}\frac{\circlearrowright}{\widetilde{N}} \\
\text { हैँ } \\
\text { L }\end{array}$ & 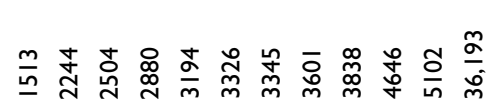 & 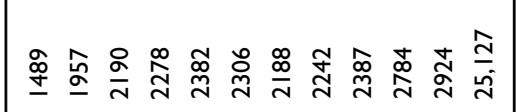 & 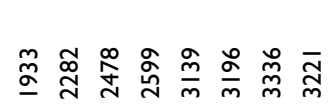 \\
\hline & & $\frac{\frac{0}{\pi}}{\Sigma}$ & 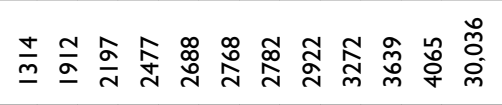 & 声 & 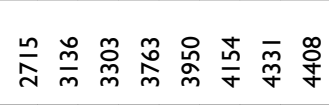 \\
\hline \multicolumn{3}{|c|}{$\frac{\square}{\square}$} & 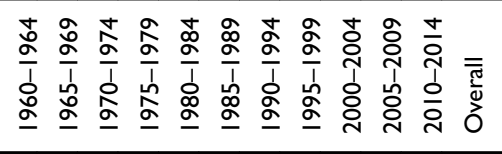 & 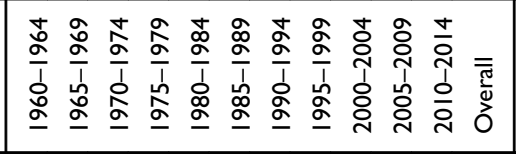 & 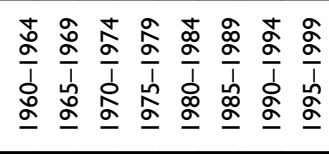 \\
\hline \multicolumn{3}{|c|}{$\stackrel{\Xi}{\sharp}$} & $\underset{\mathscr{U}}{u}$ & U & $\underset{\mathscr{E}}{\underline{\underline{E}}}$ \\
\hline
\end{tabular}




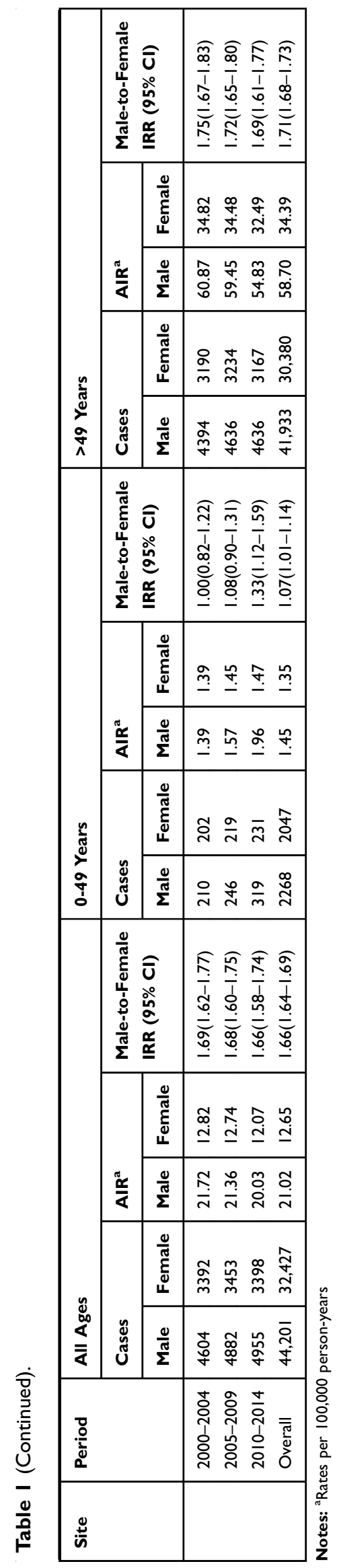

between 1970 and 1974 and the highest male-to-female IRR was 1.44 between 1995 and 1999. For rectal cancer, the lowest male-to-female IRR was 1.57 between 1980 and 1984 and the highest male-to-female IRR was 1.80 between 1975 and 1979. The male-to-female IRR of different subsites varied considerably according to the different age groups. In the younger group ( $0-49$ years), the male-tofemale IRR was 1.05 for RCC, 0.78 for LCC and 1.07 for rectal cancer. However, for CRC patients diagnosed at older ages ( $>49$ years), the male-to-female IRR was 1.05 for RCC, 1.36 for LCC, and 1.71 for rectal cancer.

For all RCC patients, the male-to-female IRR increased steadily by an average of $0.4 \%$ per year until 1995 and decreased gradually ever since by an average of $1.0 \%$ per year (Table 2 and Supplementary Figure 1). However, male-to-female IRR of the younger group increased gradually by an average of $0.7 \%$ per year throughout the period of analysis. For LCC, the overall male-to-female IRR increased gradually since 1974 by an average of $0.6 \%$ per year. For rectal cancer, the overall male-to-female IRR showed random fluctuations.

Table 3 presents the overall ASR and the temporal trends of male-to-female SRR. The ASR of CRC in females was consistently higher than males throughout the period of analysis, irrespective of anatomical subsite. The lowest male-to-female SRR of RCC was 0.81 between 1975 and 1979 and the highest male-to-female SRR was 0.96 between 1960 and 1964. For LCC, the lowest male-to -female SRR was 0.83 between 1970 and 1974 and the highest male-to-female SRR was 0.91 between 2005 and 2009. For rectal cancer, the lowest male-to-female SRR was 0.79 between 1970 and 1974 and the highest male-tofemale SRR was 0.93 between 2005 and 2009.

For RCC patients, the temporal trend of male-to-female SRR remained at a stable level during the whole study period (Table 4 and Supplementary Figure 2). For LCC patients aged younger than 50 years, male-to-female SRR increased steadily by an average of $0.6 \%$ per year. Male-to -female SRR among LCC patients aged 50 years and older remained stable during the study period. For all rectal cancer patients, male-to-female SRR increased slightly by an average of $0.2 \%$ per year, as well as patients aged 50 and older.

\section{Discussion}

Using data derived from the nationwide Swedish Cancer Registry, which covered a period of more than five decades, giving us the unique opportunity to analyze the 


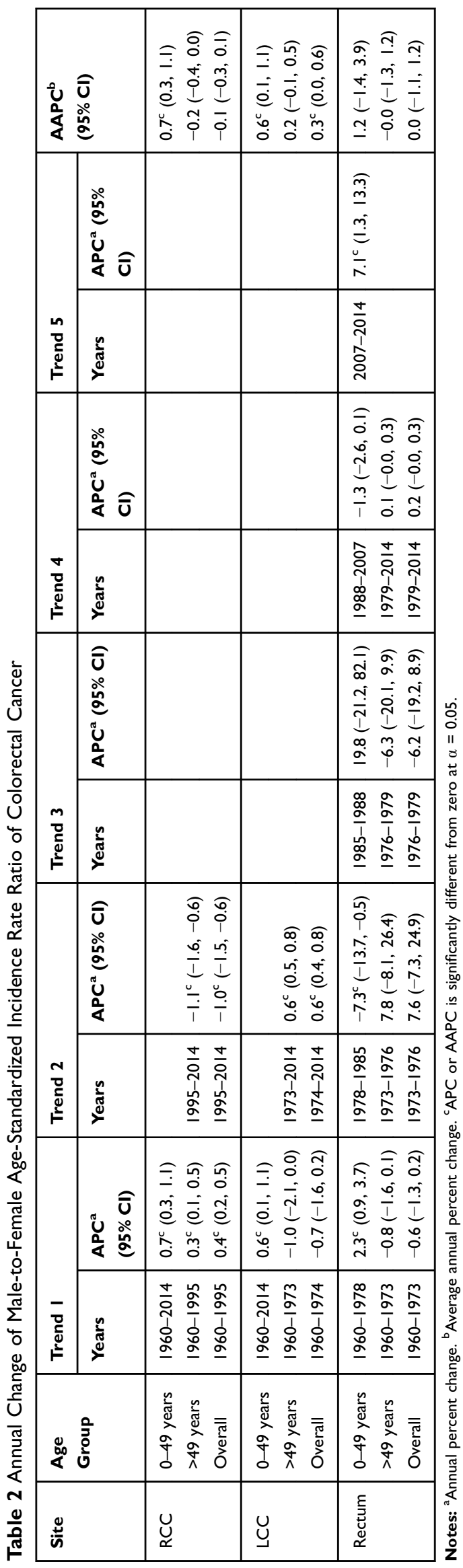

temporal trends of sex disparity in incidence and survival of CRC. Furthermore, we quantified the annual change by utilizing joinpoint regression analyses. Our results indicated that the temporal trends of sex ratio in incidence of different subtypes of CRC varied considerably during the study period and the observed sex bias varied according to the different age groups; this suggests that potential environmental factors that triggered the onset of CRC showed period-specific and age-specific patterns which may guide future studies exploring the underlying sex-specific mechanisms. In contrast, the temporal trends of sex ratio in survival of CRC remained stable during the study period.

CRC was described as an anatomical site-specific heterogeneous disease since the 1990s. ${ }^{12}$ Subsequent research has described the distinct differences in pathogenesis, genetic and epigenetic alterations, molecular pathways, immunology, and gut microbiota depending on the anatomical site of tumor. ${ }^{7-9,13}$ Environmental factors that contribute to the development of site-specific CRC were also reported. ${ }^{10,14,15}$ The explanation of the observed sex differences concerning incidence of CRC can be divided into biological and environmental mechanisms. Biological hypotheses span over anatomy structure, genetic and epigenetic, and hormonal factors. It has been reported that certain genetic and epigenetic differences between sexes may determine colorectal cancer risk. The polymorphisms of $A B C B 1$ differ significantly according to sex. ${ }^{16} \mathrm{~A}$ British study from 2003 observed a significantly increased risk of CRC in males with the $\varepsilon 2 / \varepsilon 3$ ApoE genotype. ${ }^{17} \mathrm{CpG}$ island methylator phenotype-high was associated with a higher incidence of female cecal tumors. ${ }^{18}$ A population-based case-control study revealed that estrogen exposure is a protective factor against microsatellite instability (MSI) cancer in women. ${ }^{19}$ This is reflected in that the lack of estrogen in older women increased the risk of MSI-high colon cancer. In the same study, hormone replacement therapy was associated with a reduced risk of unstable tumors.

Environmental factors include dietary, obesity, diabetes, et al. The variation of sex ratio of CRC with time period should mainly be due to sex-specific environmental exposures over time. A meta-analysis indicated that obesity was only associated with an increased risk of rectal cancer in men; this increase was not found in women. ${ }^{10}$ Meanwhile, increased body mass index (BMI) was more strongly associated with an increased risk of colon cancer than to rectal cancer. The BMI among the Swedish 


\begin{tabular}{|c|c|c|c|c|c|}
\hline & 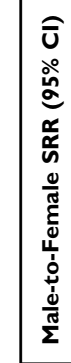 & & 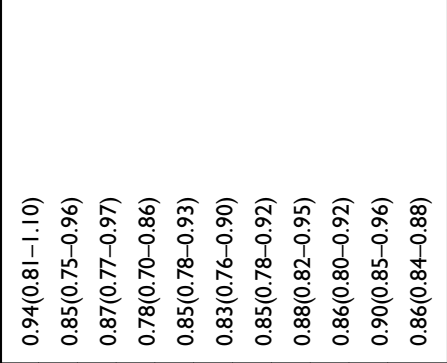 & 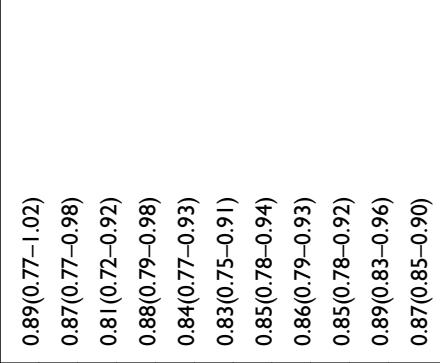 & 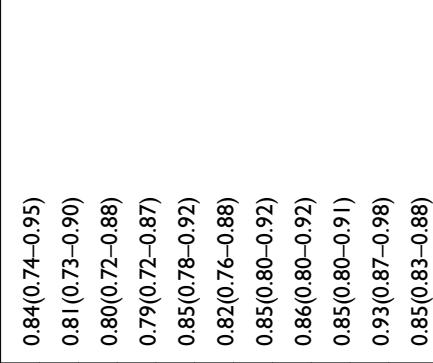 \\
\hline & 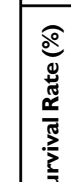 & 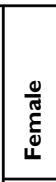 & 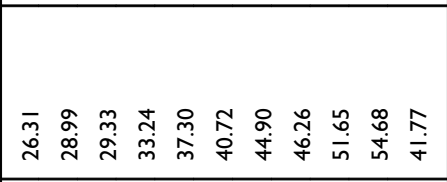 & 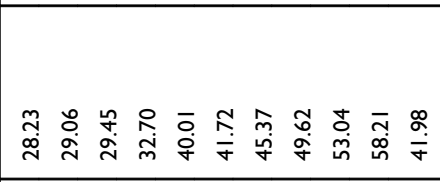 & 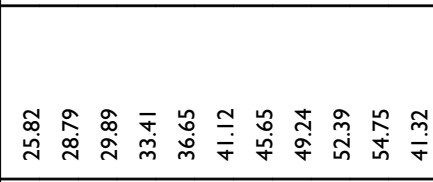 \\
\hline & 离 & $\frac{0}{\Sigma}$ & 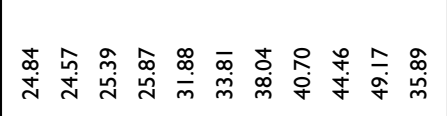 & 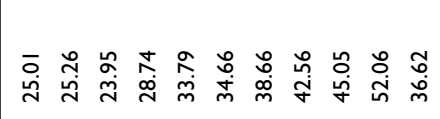 & 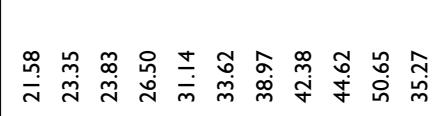 \\
\hline & 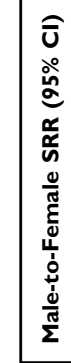 & & 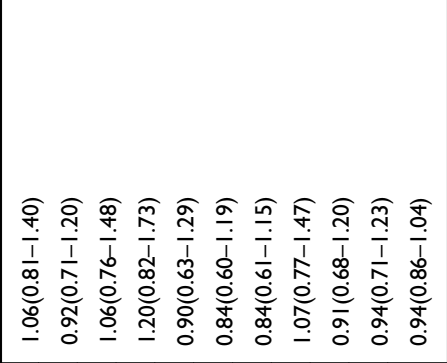 & 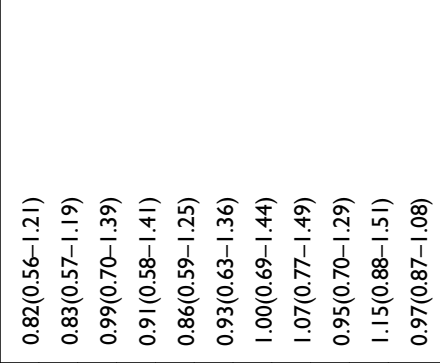 & 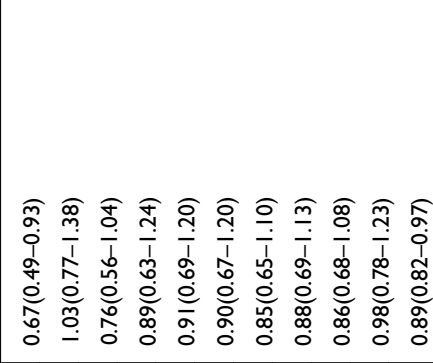 \\
\hline & 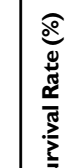 & 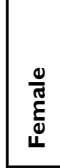 & 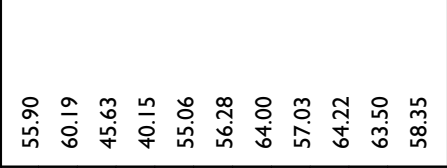 & 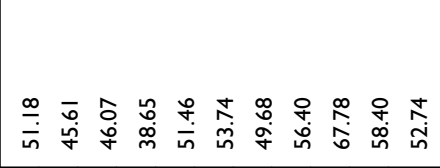 & 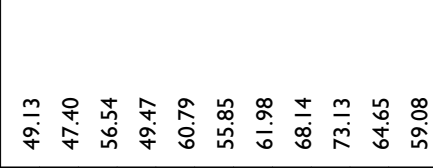 \\
\hline & ì & $\frac{\varrho}{\pi}$ & 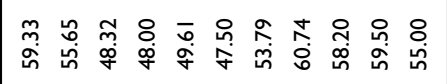 & 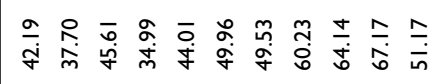 & 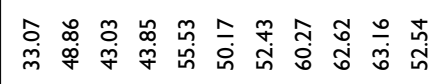 \\
\hline & 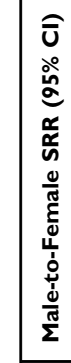 & & 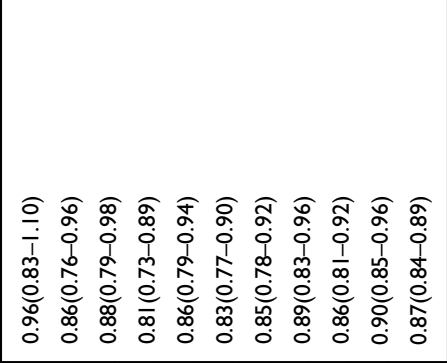 & 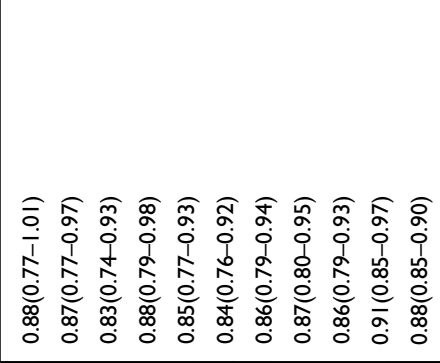 & 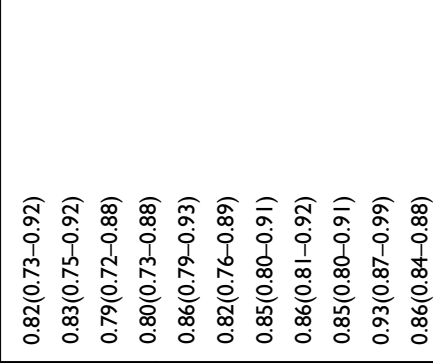 \\
\hline & 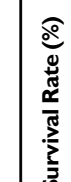 & 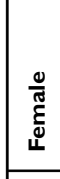 & 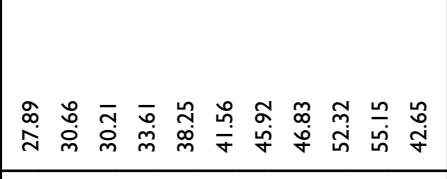 & 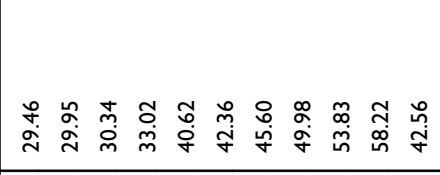 & 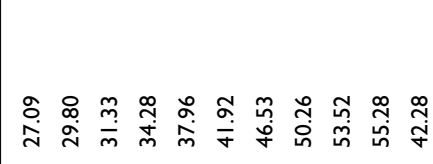 \\
\hline & 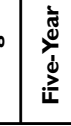 & $\frac{\omega}{\tilde{\omega}}$ & 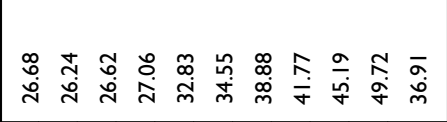 & 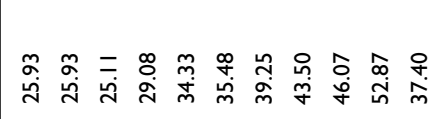 & 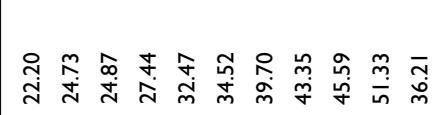 \\
\hline & & & 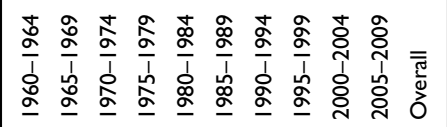 & 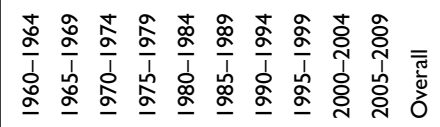 & 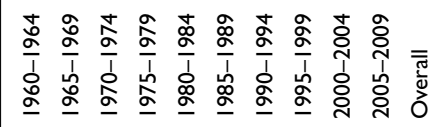 \\
\hline & & & $\underset{\check{u}}{u}$ & $\underline{u}$ & 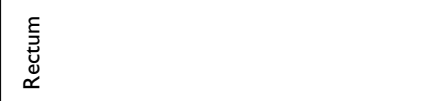 \\
\hline
\end{tabular}


Table 4 Annual Change in Male-to-Female Age-Standardized Five-Year Survival Rate Ratio of Colorectal Cancer

\begin{tabular}{|l|l|l|l|}
\hline \multirow{2}{*}{ Site } & \multirow{2}{*}{ Age Group } & \multicolumn{2}{|l|}{ Trend I } \\
\cline { 3 - 4 } & & Years & \multicolumn{2}{|l|}{ APC $^{\mathrm{a}}(\mathbf{9 5} \% \mathrm{Cl})$} \\
\hline \multirow{2}{*}{ RCC } & $0-49$ years & $1960-2009$ & $-0.1(-0.8,0.5)$ \\
& $>49$ years & $1960-2009$ & $-0.0(-0.2,0.2)$ \\
& Overall & $1960-2009$ & $-0.0(-0.2,0.1)$ \\
\hline \multirow{2}{*}{ LCC } & $0-49$ years & $1960-2009$ & $0.6^{\mathrm{b}}(0.0,1.2)$ \\
& $>49$ years & $1960-2009$ & $0.0(-0.2,0.2)$ \\
& Overall & $1960-2009$ & $0.0(-0.1,0.2)$ \\
\hline \multirow{2}{*}{ Rectum } & $0-49$ years & $1960-2009$ & $0.2(-0.3,0.8)$ \\
& $>49$ years & $1960-2009$ & $0.2^{\mathrm{b}}(0.1,0.4)$ \\
& Overall & $1960-2009$ & $0.2^{\mathrm{b}}(0.1,0.4)$ \\
\hline
\end{tabular}

Notes: annual percent change. ${ }^{b}$ APC or AAPC is significantly different from zero at $\alpha=0.05$.

population has been steadily increasing since the 1970s, especially among men and adolescents. ${ }^{20}$ Mean BMI between 1980/81 and 2004/05 increased from 24.1 to 25.5 for men and from 23.1 to 24.3 for women in Sweden. ${ }^{21}$ The association between dietary factors and CRC varied by the location of tumors and sex. High carbohydrate intake increased the risk of RCC in women, but not in men. ${ }^{22}$ Meat consumption, especially red meat, is associated with a higher risk of LCC and rectal cancer. ${ }^{23}$ The Food and Agriculture Organization of the United Nations reported that the average amount of meat consumption in Sweden increased from $76.1 \mathrm{~kg} /$ person in 2002 to $80.2 \mathrm{~kg} /$ person in 2009 , and the consumption of meat is usually higher in men than in women.

The difference in survival rates between males and females could be attributed to genetic, hormonal, and environmental factors. BRAF mutation - an independent prognostic factor for CRC - was more frequently occurring in females compared with males. ${ }^{24}$ In addition, males with LCC and rectal cancer did not benefit from adjuvant chemotherapy while females with RCC did. ${ }^{25}$ Cancer screening can reduce CRC mortality by finding cancer at an early and treatable stage. ${ }^{26}$ The five-year survival rate of CRC can reach $90 \%$ when detected at an early stage. CRC screening tests include six types of examinations; these can be divided into stool-based tests and structural examinations. ${ }^{26}$ Meanwhile, randomized clinical studies using sigmoidoscopy as the primary screening test have demonstrated both a reduced disease-specific mortality and a reduced incidence of CRC. ${ }^{27-29}$ One study showed that the prevalence of advanced LCC and rectal cancer was strongly reduced within a 10-year period after a colonoscopy procedure, but not advanced $\mathrm{RCC},{ }^{30}$ due to the fact that proximal colonic tumors are mostly flat, while distal colonic tumors are polypoid-type that are more distinguishable. In addition, a study demonstrated that colonoscopy appears to be a technically more difficult procedure in women than men. ${ }^{31}$ The rate of participating CRC screening tests is increasing for both men and women, although differences in screening use by sex have been documented. ${ }^{32} \mathrm{~A}$ study has found that men are more likely than women to receive a CRC screening test, ${ }^{2}$ which was consistent with our data that the male-to-female SRR was increasing in rectal cancer patients; this suggests the possible contribution by screening.

One weakness of this study was the lack of information on known confounding factors such as alcohol consumption, dietary data as well as screening. Meanwhile, being an ecological study, it is not possible to investigate the causal relationships between the observed temporal trends and these relevant risk factors. Our study also has several strengths. We used 55 years of CRC incidence data from the Swedish Cancer Registry that has national coverage. Thus, our analyses had sufficient statistical power to assess temporal trends of sex bias by subsites and guarantee the accuracy of this study.

\section{Conclusion}

In conclusion, we found that the temporal trend of CRC showed a different pattern and the sex disparity of RCC, LCC, and rectal cancer varied by age at onset. The temporal variations of sex bias of CRC might be related to sex-specific exposures of environmental risk factors. Further understanding of the effect of modifiable environmental factors on CRC and sex-related exposure factors will be of vital importance. Although male sex is still associated with poorer survival, the gap of sex ratio of survival narrowed gradually, especially for rectal cancer.

\section{Acknowledgments}

The authors wish to thank the CPF's science editor Patrick Reilly for his valuable comments on the text. This work was supported by grants awarded to Dr. Jianguang Ji by the Swedish Research Council (2016-02373) and Cancerfonden (2017 CAN2017/340) and The Crafoord Foundation, to Professor Kristina Sundquist and to Professor Jan Sundquist by the Swedish Research Council (2018-02400 and 2016-01176, respectively), to Jan Sundquist, Kristina Sundquist, and Jianguang Ji by ALF funding from Region Skåne. The funding agencies had no role in the design and conduct of the study; in the 
collection, analysis, and interpretation of the data; or in the preparation, review, or approval of the manuscript. The researchers were independent of the funding agencies.

\section{Disclosure}

The authors report no conflicts of interest in this work.

\section{References}

1. Bray F, Ferlay J, Soerjomataram I, Siegel RL, Torre LA, Jemal A. Global cancer statistics 2018: GLOBOCAN estimates of incidence and mortality worldwide for 36 cancers in 185 countries. CA Cancer J Clin. 2018;68(6):394-424. doi:10.3322/caac.v68.6

2. Kim SE, Paik HY, Yoon H, Lee JE, Kim N, Sung MK. Sex- and gender-specific disparities in colorectal cancer risk. World $J$ Gastroenterol. 2015;21(17):5167-5175. doi:10.3748/wjg.v21. i17.5167

3. Hendifar A, Yang D, Lenz F, et al. Gender disparities in metastatic colorectal cancer survival. Clin Cancer Res. 2009;15(20):6391-6397. doi:10.1158/1078-0432.CCR-09-0877

4. Balasubramanian SP. Gender differences in long-term survival of patients with colorectal cancer. Br J Surg. 2002;89(4):489. doi:10.1046/j.1365-2168.2002.20882.x

5. Yang Y, Wang G, He J, et al. Gender differences in colorectal cancer survival: a meta-analysis. Int J Cancer. 2017;141(10):1942-1949. doi:10.1002/ijc.v141.10

6. Lee GH, Malietzis G, Askari A, Bernardo D, Al-Hassi HO, Clark SK. Is right-sided colon cancer different to left-sided colorectal cancer? A systematic review. Ejso-Eur J Surg Onc. 2015;41(3):300-308. doi:10.1016/j.ejso.2014.11.001

7. Glebov OK, Rodriguez LM, Nakahara K, et al. Distinguishing right from left colon by the pattern of gene expression. Cancer Epidem Biomar. 2003;12(8):755-762.

8. Iacopetta B, Heyworth J, Girschik J, Grieu F, Clayforth C, Fritschi L. The MTHFR C677T and DeltaDNMT3B C-149T polymorphisms confer different risks for right- and left-sided colorectal cancer. Int $J$ Cancer. 2009;125(1):84-90. doi:10.1002/ijc.24324

9. Gill SR, Pop M, DeBoy RT, et al. Metagenomic analysis of the human distal gut microbiome. Science. 2006;312(5778):1355-1359. doi:10.1126/science. 1124234

10. Larsson SC, Wolk A. Obesity and colon and rectal cancer risk: a meta-analysis of prospective studies. Am J Clin Nutr. 2007;86 (3):556-565. doi:10.1093/ajcn/86.3.556

11. Ji J, Sundquist K, Sundquist J, Hemminki K. Comparability of cancer identification among death registry, cancer registry and hospital discharge registry. Int J Cancer. 2012;131(9):2085-2093. doi:10.1002/ ijc. 27462

12. Bufill JA. Colorectal cancer: evidence for distinct genetic categories based on proximal or distal tumor location. Ann Intern Med. 1990;113(10):779-788. doi:10.7326/0003-4819-113-10-779

13. Paski SC, Wightman R, Robert ME, Bernstein CN. The importance of recognizing increased cecal inflammation in health and avoiding the misdiagnosis of nonspecific colitis. Am J Gastroenterol. 2007;102 (10):2294-2299. doi:10.1111/ajg.2007.102.issue-10

14. Chao A, Thun MJ, Connell CJ, et al. Meat consumption and risk of colorectal cancer. JAMA. 2005;293(2):172-182. doi:10.1001/jama. 293.2.172

15. Mills KT, Bellows CF, Hoffman AE, Kelly TN, Gagliardi G. Diabetes mellitus and colorectal cancer prognosis: a meta-analysis. Dis Colon Rectum. 2013;56(11):1304-1319. doi:10.1097/DCR.0b 013e3182a479f9
16. Martinelli M, Scapoli L, Cura F, et al. Colorectal cancer susceptibility: apparent gender-related modulation by ABCB1 gene polymorphisms. J Biomed Sci. 2014;21:89.

17. Watson MA, Gay L, Stebbings WS, Speakman CT, Bingham SA, Loktionov A. Apolipoprotein E gene polymorphism and colorectal cancer: gender-specific modulation of risk and prognosis. Clin Sci (Lond). 2003;104(5):537-545. doi:10.1042/CS20020329

18. Bae JM, Kim JH, Cho NY, Kim TY, Kang GH. Prognostic implication of the $\mathrm{CpG}$ island methylator phenotype in colorectal cancers depends on tumour location. Br J Cancer. 2013;109(4):1004-1012. doi: $10.1038 /$ bjc. 2013.430

19. Slattery ML, Potter JD, Curtin K, et al. Estrogens reduce and withdrawal of estrogens increase risk of microsatellite instability-positive colon cancer. Cancer Res. 2001;61(1):126-130.

20. Ezzati M, Bentham J, Di Cesare M, et al. Worldwide trends in body-mass index, underweight, overweight, and obesity from 1975 to 2016: a pooled analysis of 2416 population-based measurement studies in 128.9 million children, adolescents, and adults. Lancet. 2017;390(10113):2627-2642.

21. Caman OK, Calling S, Midlov P, Sundquist J, Sundquist K, Johansson SE. Longitudinal age-and cohort trends in body mass index in Sweden-a 24-year follow-up study. BMC Public Health. 2013;13:893. doi:10.1186/1471-2458-13-893

22. Borugian MJ, Sheps SB, Whittemore AS, Wu AH, Potter JD, Gallagher RP. Carbohydrates and colorectal cancer risk among Chinese in North America. Cancer Epidem Biomar. 2002;11 (2):187-193.

23. Larsson SC, Wolk A. Meat consumption and risk of colorectal cancer: a meta-analysis of prospective studies. Int $J$ Cancer. 2006;119(11):2657-2664. doi:10.1002/(ISSN)1097-0215

24. Roth AD, Tejpar S, Delorenzi M, et al. Prognostic role of KRAS and BRAF in stage II and III resected colon cancer: results of the translational study on the PETACC-3, EORTC 40993, SAKK 60-00 trial. J Clin Oncol. 2010;28(3):466-474. doi:10.1200/JCO.20 09.23 .3452

25. Elsaleh H, Joseph D, Grieu F, Zeps N, Spry N, Iacopetta B. Association of tumour site and sex with survival benefit from adjuvant chemotherapy in colorectal cancer. Lancet. 2000;355 (9217):1745-1750. doi:10.1016/S0140-6736(00)02261-3

26. Wolf AMD, Fontham ETH, Church TR, et al. Colorectal cancer screening for average-risk adults: 2018 guideline update from the American Cancer Society. CA Cancer J Clin. 2018;68(4):250-281. doi: $10.3322 /$ caac. 21457

27. Atkin WS, Cook CF, Cuzick J, et al. Single flexible sigmoidoscopy screening to prevent colorectal cancer: baseline findings of a UK multicentre randomised trial. Lancet. 2002;359(9314):1291-1300.

28. Schoen RE, Pinsky PF, Weissfeld JL, et al. Colorectal-cancer incidence and mortality with screening flexible sigmoidoscopy. $N$ Engl J Med. 2012;366(25):2345-2357. doi:10.1056/NEJMoa1114635

29. Lee SJ, Boscardin WJ, Stijacic-Cenzer I, Conell-Price J, O’Brien S, Walter LC. Time lag to benefit after screening for breast and colorectal cancer: meta-analysis of survival data from the United States, Sweden, United Kingdom, and Denmark. BMJ. 2013;346:e8441. doi:10.1136/bmj.e8441

30. Brenner H, Hoffmeister M, Arndt V, Stegmaier C, Altenhofen L, Haug U. Protection from right- and left-sided colorectal neoplasms after colonoscopy: population-based study. J Natl Cancer Inst. 2010;102(2):89-95. doi:10.1093/jnci/djp436

31. Saunders BP, Fukumoto M, Halligan S, et al. Why is colonoscopy more difficult in women? Gastrointest Endosc. 1996;43(2):124-126. doi:10.1016/S0016-5107(06)80113-6

32. Meissner HI, Breen N, Klabunde CN, Vernon SW. Patterns of colorectal cancer screening uptake among men and women in the United States. Cancer Epidem Biomar. 2006;15(2):389-394. doi:10.1158/ 1055-9965.EPI-05-0678 


\section{Publish your work in this journal}

Clinical Epidemiology is an international, peer-reviewed, open access, online journal focusing on disease and drug epidemiology, identification of risk factors and screening procedures to develop optimal preventative initiatives and programs. Specific topics include: diagnosis, prognosis, treatment, screening, prevention, risk factor modification,

Submit your manuscript here: https://www.dovepress.com/clinical-epidemiology-journal systematic reviews, risk \& safety of medical interventions, epidemiology \& biostatistical methods, and evaluation of guidelines, translational medicine, health policies \& economic evaluations. The manuscript management system is completely online and includes a very quick and fair peer-review system, which is all easy to use. 